\title{
There Are Many Reasons to Drive a BMW: Does Imagined Ease of Argument Generation Influence Attitudes?
}

\author{
MICHAELA WÄNKE \\ GERD BOHNER \\ ANDREAS JURKOWITSCH*
}

\begin{abstract}
The effects of imagined versus actual ease of self-generating product-related information were investigated. An ad invited recipients to name either one reason or 10 reasons for (against) choosing a BMW over a Mercedes. Participants who complied with the task experienced the retrieval of one reason as easier than the retrieval of 10 reasons. Participants who did not comply nevertheless imagined the former as easier than the latter. Independent of whether ease was actually experienced or merely imagined, participants evaluated BMW more (less) favorably and Mercedes less (more) favorably when the retrieval was easy rather than difficult.
\end{abstract}

\begin{abstract}
A classic notion in the persuasion literature holds that arguments that are self-generated by the targets of influence are especially effective in attitude change (see, e.g., Janis and King 1954; King and Janis 1956). In product communication, a frequently used strategy of exploiting this phenomenon is to let consumers generate relevant brand information.

However, recent research suggests that the effectiveness of self-generation may be moderated by the experienced ease or difficulty with which relevant material comes to mind. For example, in a study by Wänke, Bless, and Biller (1996), participants listed either three or seven self-generated reasons why to use ("pro") or why not to use ("contra") public transportation. Listing three arguments felt easy, while listing seven arguments felt difficult. As a result, participants who had listed seven pro reasons reported less favorable attitudes toward the issue than participants who had listed only three pro reasons. Conversely, listing seven contra reasons led to more favorable judgments than listing three contra reasons. Other
\end{abstract}

* Michaela Wänke is research associate at the Psychologisches Institut, Universität Heidelberg, Hauptstr. 47-51, D-69117 Heidelberg, Germany; e-mail: ziv7@ @si-sv1.psi.uni-heidelberg.de. Gerd Bohner is assistant professor of social psychology, and Andreas Jurkowitsch is a diploma student in sociology; both are at the Lehrstuhl für Sozialpsychologie, Universität Mannheim, D-68131 Mannheim, Germany. Preparation of the manuscript was supported by grant Bl 289/5-1 from the Deutsche Forschungsgemeinschaft to H. Bless, N. Schwarz, and M. Wänke, and grant Schw 278/5 to N. Schwarz, G. Bohner, and H. Bless. The authors gratefully acknowledge comments by the editor, associate editor, and several reviewers. participants who had not generated arguments themselves but were exposed to the arguments the former participants had generated showed the reverse effect. These mere receivers of arguments were more persuaded by seven than by three arguments. These studies suggest that experiencing difficulty in generating supportive arguments or counterarguments may reduce or, depending on the strength of the effect, even reverse the impact of their valence on attitude judgments (for similar findings on judgments regarding the self, see Schwarz et al. [1991b]).

Individuals seem to derive informational input from the experienced ease or difficulty with which relevant material comes to mind. A number of explanations for this effect seem possible (for a detailed discussion, see Wänke et al. [1996]). According to the availability heuristic, difficulty may lead individuals to infer that the amount of information is rather limited (Tversky and Kahneman 1982; Wänke, Schwarz, and Bless 1995). That is, from the lack of positive information they may derive a negative judgment. Alternatively, the lack of informational evidence may cause individuals to use the information conservatively or to correct for its implications (Wänke and Gierke 1995). Other evidence suggests that retrieval difficulty may result in perceiving the quality of the information as uncompelling (Werrmann 1996), which may also contribute to a reduced impact.

On the basis of these results regarding the moderating effect of subjective experience in information retrieval, we may assume that an ad prompting consumers to retrieve supportive arguments by themselves is successful only if consumers experience the task as easy. However, the available studies differ from a typical advertising situ- 
ation in many respects. Thus, it is unclear whether inferences based on these studies are valid in an advertising context. First, in extant studies, participants were asked to write down the generated arguments, whereas ads ask consumers only to think of relevant benefits. Second, in these studies, careful control was exercised to make sure that participants in the difficult condition actually completed the task even though it was difficult. In a realistic setting of ad exposure, consumers may simply stop generating product benefits once the task turns difficult. Thus, they may never actually experience difficulty. In addition, the short time span and low involvement that consumers usually devote to the processing of advertising may in fact interfere with any argument generation at all. Thus, the question remains as to what extent ease or difficulty in generating product benefits in response to an ad may play a role in product evaluation.

It has been suggested, however, that "it is not necessary to perform the actual operations of retrieval. . . . It suffices to assess the ease with which these operations could be performed" (emphasis added; Tversky and Kahneman 1982, p. 164). In other words, consumers' anticipation that the retrieval of product benefits will be difficult may be sufficient to elicit an unfavorable inference about the product. The notion of anticipated difficulty or imagined difficulty as an elicitor of the inferences triggered by actual experiences, though untested so far, seems plausible for several reasons. The ability of individuals to judge the difficulty of tasks before they begin them seems highly functional. An estimation of the difficulty would allow appropriate allocation of resources to the task, judgment of the rate of success, and an assessment of the time needed for completion. In addition, it seems unlikely that such a system evolved without the estimates being functionally equivalent to actual experiences. Indeed, evidence from other experiential domains has shown that merely imagining experiences suffices to induce the corresponding mood states (see, e.g., Strack, Schwarz, and Gschneidinger 1985).

The notion that imagined ease or difficulty may trigger the same effects as the actual experience is particularly interesting for advertising purposes. Because ad recipients usually devote limited attention to the processing of ad information, consumers frequently may not engage in information generation. Nevertheless, ads that ask consumers to do just that may be effective by way of imagined information retrieval. This perspective poses a shift regarding the necessary conditions of how available information influences attitudinal judgments. The classical assumption in social cognition (for reviews, see Higgins [1989]; Wyer and Srull [1989]) and consumer cognition (see, e.g., Kisielius and Sternthal [1984]; for a review, see Kardes [1994]) suggests that judgments reflect the content of the information that is accessible at the time of judgment. According to much recent work, such as described earlier by Schwarz, Wänke, and their colleagues (Schwarz et al. 1991b; Wänke et al. 1996), the impact of the content of accessible information is moderated by the subjectively experienced ease of accessibility. Although judgments reflected the valence of information for which retrieval was experienced as easy, this was less the case for information for which retrieval was experienced as difficult.

In the present article, we develop this perspective one step further and argue that, rather than actual experience, the mere anticipation of how easy or difficult it would be to retrieve relevant information may suffice to affect the judgment. Thus, a judgment requires neither access to actual information content nor any actual experience accompanying the retrieval process; rather, the cognitive appraisal of the ease of retrieving particular information may suffice.

\section{Goals of the Study}

Our study has two purposes. One is to test the moderating role of ease in an advertising context as described above. Within this framework, our second goal is to investigate whether anticipated ease is functionally equivalent to actually experienced ease, as proposed by Tversky and Kahneman (1982). Support for our hypotheses would suggest an interesting twist to the traditional persuasive communication in advertising, namely, that positive attitude change can be enhanced by inducing difficulty in retrieving negative arguments. Thus, it may be worthwhile for a campaign to ask consumers to generate negative arguments. If it can be ensured that consumers experience or at least anticipate difficulty at this task, they may actually develop a more positive attitude toward the product.

\section{Overview and Hypotheses}

In our study, participants were presented with an ad that proposed that recipients should generate either one or 10 reasons why one should drive a BMW or why one should not drive a BMW. We assumed that in the onereason condition participants would actually retrieve one reason, experiencing ease. For the 10-reasons condition, we assumed that participants would either $(a)$ experience failure or high difficulty in attempting retrieval or $(b)$ anticipate to experience difficulty or failure and thus decide not to engage in retrieval at all. Comparing the two groups of participants (described by $a$ and $b$ ) would shed light on the untested proposition that actual and anticipated ease are functionally equivalent.

The ads were presented as comparative BMW versus Mercedes ads. We chose these two brands because they are the major competitors in the market of upper-middleclass and luxury cars in Germany and are often seen as substitutes for each other, much like the Coke and Pepsi rivalry in the United States. Presenting comparative ads allowed us to test our hypothesis regarding the retrieval of negative information, which otherwise may not have seemed credible.

We expected that ease would moderate the impact of information content on brand evaluations and preferences. More specifically, we expected that in the one-reason condition, ad recipients who are asked to think of a pro 
reason would evaluate BMW more positively than recipients who are asked to think of a contra reason, because both groups would use the accessible information. In the 10-reason condition, this difference between ads asking to generate pro reasons and those asking to generate contra reasons should be diminished, because retrieval difficulty - experienced or anticipated - would interfere with the impact that the implications of the ad content would otherwise have. For attitudes toward BMW's competitor Mercedes-Benz, we expected the opposite impact of argument valence. ${ }^{1}$ That is, ad recipients should arrive at more favorable attitudes toward Mercedes after retrieving contra-BMW reasons than after retrieving pro-BMW reasons. Again, retrieval difficulty should weaken the influence of information content, resulting in diminished differences between the ads requesting pro reasons and those requesting contra reasons in the 10-reason condition.

Pilot studies with respondents from the same population as used in the main study had shown that both brands are evaluated favorably, with a slight advantage for BMW. Applying this observation to the above predictions, we expected BMW's advantage to be affected by the content of retrieved information and the ease of this retrieval. BMW's advantage over Mercedes should be more pronounced when the ad asked for one pro-BMW reason than when it asked for one contra-BMW reason, but this difference should diminish for the retrieval of 10 pro and contra reasons.

\section{METHOD}

\section{Participants and Design}

One hundred sixty male business students at the University of Mannheim voluntarily participated in the study. Participants were randomly assigned to the conditions of a 2 (argument generation task: easy vs. difficult) $\times 2$ (valence of arguments: pro vs. contra) $\times 2$ (order of dependent measures: BMW rating first vs. Mercedes rating first) factorial design. The latter variation was introduced to control for possible order effects.

\section{Stimulus Materials}

Four different advertisements were created. Valence of the to-be-retrieved material was varied by using ads that either asked for the retrieval of reasons why to choose a BMW or asked for reasons why not to choose a BMW. Within each of these valence conditions, ease of retrieval was varied by the number of reasons to be retrieved: the

\footnotetext{
${ }^{1}$ In principle, it may also be possible, however, that the evaluation of BMW would be transferred to Mercedes because of the similarity of the two brands. This would likely be the case if the arguments retrieved were generalizable to the whole category of luxury cars, e.g., "waste energy,', "look nouveau riche,'” etc. A comparative ad, however, should induce subjects to think in terms of distinctive advantages or disadvantages of the respective brand compared to the competitor. We therefore did not expect such generalization in the present experiment.
}

easy version asked participants to retrieve one reason, whereas the difficult version asked participants to retrieve 10 reasons. Depending on experimental condition, the ads read: "There are many reasons (not) to choose a BMW. Can you name one (10)?' Each ad contained a headline saying "BMW or Mercedes?', This headline was created to present the ad as comparative advertising in order to make our negative valence condition appear more plausible.

To rule out the possibility that the ads evoked differences in liking that might pose an alternative explanation for the predicted effects, we ran an independent study. In a between-subjects design, 40 participants evaluated one of the four ads, indicating how much they liked it and how "'annoying," "'witty,', "'well done,' "'clever,', and "convincing" they considered it to be. We found no differences (all $F$ 's $<1$ ). Moreover, we asked these participants to indicate for which brand they thought the ad was. Again, the presumed brand did not differ between the ads $(F<1)$, with about half of the participants attributing each of the ads to either brand.

In addition to the target ads, four filler ads were presented that also featured comparative ads (lifestyle magazines) or ads in which two brands advertised their complementary function (sunglasses and jeans; champagne and aspirin; frying pan and frozen fish). All filler ads consisted only of a slogan (e.g., "Where do Iglo fish fingers feel best? In Fissler frying pans, of course' '). ${ }^{2}$

\section{Procedure and Dependent Variables}

The experiment was run in groups with four to six participants. The sessions were randomly assigned to the experimental conditions of ease and valence. Both order conditions were distributed randomly in each session. Participants were recruited for participation in a study on the evaluation of advertising. They were asked to read newly created slogans for various products and later to answer some questions. All ads were projected by an overhead projector. Each ad was presented for 10 seconds. The target ad was the third ad in a series of five ads.

After the last ad was shown, the experimenter distributed questionnaires that contained the dependent measures, which participants completed at their own pace. Depending on the order condition, participants gave a general evaluation and purchase interest for BMW followed by the respective ratings for Mercedes, or vice versa. They then stated their preference for BMW versus Mercedes. All ratings were made on a graphic rating scale consisting of seven equal-sized boxes with labeled end points. The scale ends were labeled "highly favorable", and "highly unfavorable" for the brand evaluations, "very high" and "very low" for purchase interest, and "BMW' and "Mercedes"' for the direct preference. After this task, participants' immediate reaction to the ad was assessed. They indicated whether they had thought

\footnotetext{
${ }^{2}$ Iglo and Fissler are real brands in the respective product categories.
} 
they could think of one $(10)$ reasons $(1=$ "Yes, I'm absolutely confident I can retrieve one [10] reason[s]', $7=$ 'No, I'm absolutely confident that I cannot retrieve one [10] reason[s]''). Moreover, participants indicated with a yes or no response whether they had actually tried to retrieve reasons. This question served to divide participants into those that had actual retrieval experiences and those that had only anticipated retrieval experiences. The former also indicated on a seven-point scale (with end points "very easy" and "very difficult") how easy or difficult this retrieval felt. Finally, those respondents who had retrieved reasons were asked to list those reasons.

\section{RESULTS}

\section{Manipulation Checks}

Respondents in the easy (vs. difficult) condition were more confident that they could retrieve the requested number of reasons $(\bar{X}=2.6$ vs. $\bar{X}=5.6, F(1,152)=186.63$, $p<.001$; all other effects $p>.16)$. In the easy condition, 49 percent of the participants actually attempted to retrieve one reason, but only 30 percent began to retrieve reasons in the difficult condition $\left(\chi^{2}(1)=5.89, p<.05\right)$. Although not predicted by any hypothesis, participants in the pro condition (47 percent) were more likely to search for arguments than participants in the contra condition (31 percent; $\left.\chi^{2}(1)=4.42, p<.05\right)$. Those participants that actually retrieved arguments found this task easier in the easy condition than in the difficult condition $(\bar{X}=3.3$ vs. $\bar{X}=4.4, F(1,55)=7.02, p<.02)$.

We had initially expected that all participants in the easy condition would attempt to retrieve information and that comparing actual and anticipated experience would only be possible for the difficult condition. As it turned out, only half of the participants in the easy condition actually attempted to retrieve information. This enabled us to compare the effects of experienced versus imagined ease or difficulty in both conditions by using attempted retrieval (yes $[N=63]$ vs. no $[N=97]$ ) as a factor in the following analyses of brand evaluation and preference.

\section{Brand Evaluations}

The graphic rating scales were coded as seven-point rating scales, with higher numbers reflecting a more positive attitude or purchase interest. An ANOVA with these scores using ease, valence, order, and attempted retrieval as between-subjects factors and brand as a within-subjects factor revealed the same pattern of findings for the attitude scale and the purchase interest scale. ${ }^{3}$ Because of the relatively high correlation between the two scales $(r$ $=.63$ for BMW and $r=.57$ for Mercedes), they were averaged into a compound score. ${ }^{4}$ The same ANOVA was then conducted with the compound score, yielding a

\footnotetext{
${ }^{3} \mathrm{~A}$ regression approach was applied in order to take unequal cell sizes into account.

${ }^{4}$ All reported interactions were also significant on the item level.
}

significant three-way interaction of ease, valence, and brand $(F(1,144)=52.07, p<.001)$. All effects of equal or higher order were nonsignificant $(F<1.32, p>.25)$. As Figure 1 reveals, the combination of ease and valence resulted in oppositely directed effects on the evaluation of BMW and Mercedes. Univariate analyses with ease, valence, order, and attempted retrieval as the independent factors revealed that the crossover interactions of valence and ease were significant for the evaluation of BMW $(F(1,144)=43.06, p<.001)$ and for the evaluation of Mercedes $(F(1,144)=11.05, p<.001)$.

The fact that experienced versus anticipated retrieval did not interact with the other factors suggests that the impact of experienced and anticipated ease is similar. In fact, separate analyses document that the predicted threeway interactions between ease, valence, and brand are significant for actual experience $(F(1,144)=17.04$, $p<.001)$ and for anticipated experience $(F(1,144)$ $=42.56, p<.001$; see Table 1 for the means and univariate ANOVAs). ${ }^{5}$

The brand evaluations depicted in Figure 1 were further analyzed for simple comparisons.

BMW Evaluation. On the basis of a one-way ANOVA with the ad type as the independent variable $(F(3,156)=16.92, p<.001)$, simple comparisons were tested by a one-tailed modified least significant difference test. As predicted, participants evaluated BMW more positively when the ad suggested they retrieve one reason for choosing a BMW rather than retrieve one reason against choosing a BMW $(p<.001)$. Furthermore, as predicted, in the pro-BMW condition the evaluation became less favorable for 10 reasons $(p<.001)$, while in the contraBMW condition the evaluation became more favorable in case of 10 reasons $(p<.001)$. This countervalence effect of the ad that asked for 10 reasons was so strong that the impact of valence not only diminished but reversed. Participants reported less favorable attitudes toward BMW following an ad that suggested they retrieve 10 pro reasons as compared to one that suggested they retrieve 10 contra reasons $(p<.001)$.

Mercedes Evaluation. The effects of the different ads on the evaluation of Mercedes $(F(3,156)=5.48$, $p<.001$ for the one-way ANOVA) were generally smaller than the effects on the evaluation of BMW. Evaluations for Mercedes following ads that suggested the retrieval of one reason for or against BMW did not differ significantly from each other. However, according to expectations, compared with the respective one-reason conditions, evaluations for Mercedes became more favorable when 10 pro-BMW reasons were requested $(p<.02)$ and less favorable when 10 contra-BMW reasons were requested $(p<.03)$. In fact, ads suggesting the retrieval of 10 reasons led to significantly more positive Mercedes

\footnotetext{
${ }^{5}$ Mean square (error) for testing the effects within experienced versus anticipated retrieval was computed from the full design; hence $d f$ (error) = 144 (see Rosenthal and Rosnow 1985).
} 
FIGURE 1

MEAN EVALUATION OF BMW AND MERCEDES AS A FUNCTION OF AD TYPE

\section{Evaluation of BMW}

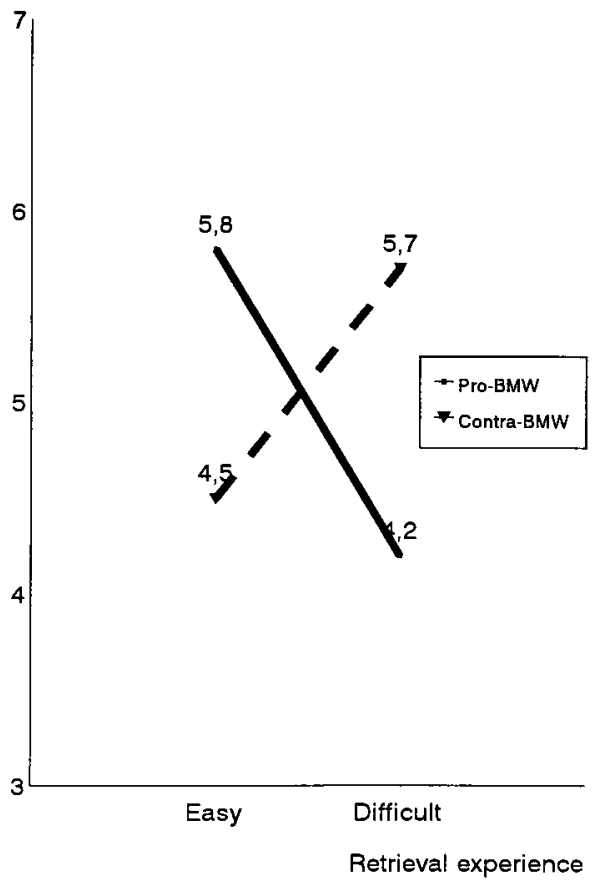

Evaluation of Mercedes

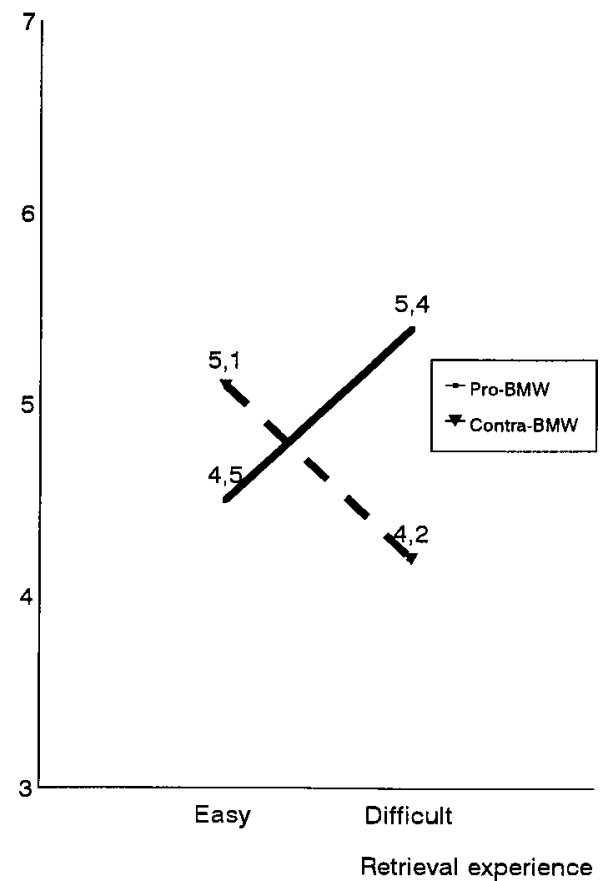

TABLE 1

MEAN EVALUATION OF BMW AND MERCEDES AND DIRECT PREFERENCES AS A FUNCTION OF AD TYPE AND ACTUAL VERSUS ANTICIPATED EXPERIENCE

\begin{tabular}{|c|c|c|c|c|c|}
\hline & \multicolumn{4}{|c|}{ Ad type } & \multirow[b]{3}{*}{ Ease $\times$ valence } \\
\hline & \multicolumn{2}{|c|}{ Easy } & \multicolumn{2}{|c|}{ Difficult } & \\
\hline & Pro BMW & Contra BMW & Pro BMW & Contra BMW & \\
\hline \multicolumn{6}{|l|}{ Actual experience: } \\
\hline$N=63$ & $N=22$ & $N=17$ & $N=16$ & $N=8$ & \\
\hline Evaluation of BMW & 5.6 & 4.7 & 4.4 & 5.9 & $F(1,144)=11.56, p<.001$ \\
\hline Evaluation of Mercedes & 4.6 & 5.2 & 5.7 & 4.5 & $F(1,144)=4.88, p<.05$ \\
\hline Direct preference & 1.0 & -.1 & -.6 & 1.1 & $F(1,152)=10.28, p<.01$ \\
\hline \multicolumn{6}{|l|}{ Anticipated experience: } \\
\hline$N=97$ & $N=18$ & $N=23$ & $N=24$ & $N=32$ & \\
\hline Evaluation of BMW & 6.2 & 4.4 & 4.1 & 5.7 & $F(1,144)=40.80, p<.001$ \\
\hline Evaluation of Mercedes & 4.5 & 5.0 & 5.2 & 4.2 & $F(1,144)=6.72, p<.02$ \\
\hline Direct preference & 1.3 & -.4 & -.8 & 1.5 & $F(1,152)=34.10, p<.001$ \\
\hline
\end{tabular}

NOTE. - Brand evaluation scales range from 1 to 7 , and higher ratings reflect a more positive evaluation of the respective target brand. Direct preferences scales range from -3 to +3 , and positive numbers reflect a relative preference for BMW.

evaluations when the reasons were for rather than against BMW $(p<.01)$.

Comparisons between Brands. On the basis of pilot studies, we had expected an advantage for BMW over Mercedes. However, we had expected the size of the advantage to be moderated by the experimental conditions.
Differences between brands were tested by subtracting the Mercedes evaluation from the BMW evaluation for each participant and submitting the difference score to one-way ANOVA with ad type as the independent variable $(F(3,156)=21.85, p<.001)$. On the basis of this, a modified least significant difference test was conducted. These comparisons revealed that an ad that asked why to 
choose a BMW led to an advantage for BMW over Mercedes only if one reason was requested but not if 10 reasons were requested $(p<.001)$. In fact, in the latter condition, BMW was rated less favorably than Mercedes. For reasons why not to choose a BMW, Mercedes enjoyed an advantage over BMW when one reason was requested but not when 10 reasons were requested $(p<.001)$. Again, a reversal emerged and BMW was rated more favorably than Mercedes in the latter condition.

\section{Preference}

The ratings were coded so that +3 reflects a preference for BMW and -3 a preference for Mercedes. The direct preference ratings reflected that preference was strongly influenced by type of ad, as Figure 2 indicates. An ANOVA with ease, valence, and attempted retrieval as between-subjects factors revealed a significant two-way interaction of ease and valence $(F(1,152)=37.21, p$ $<.001)$. All effects of equal or higher order were nonsignificant $(F<1.03, p>.32)$. The pattern was significant for actual and for anticipated retrieval (see Table 1).

Simple comparisons, according to a one-tailed modified least significant difference test based on a one-way ANOVA with ad type as a factor $(F(3,156)=16.91$, $p<.001)$, showed a higher preference for BMW in response to the ad that asked for one reason why to choose a BMW compared to why not to choose a BMW ( $p$ $<$.001). Likewise, and in line with our prediction, preference for BMW was lower following an ad asking for 10 pro-BMW reasons rather than only one $(p<.001)$. But preference for BMW was higher following an ad asking for 10 contra-BMW reasons rather than only one ( $p$ $<.001)$. Again, the impact of difficulty in information retrieval was strong enough to reverse the effect of valence, so that participants preferred BMW more when the ad asked for 10 reasons why not to choose a BMW rather than 10 reasons why to choose a BMW $(p<.001)$.

\section{DISCUSSION}

Our hypotheses were supported. Whereas previous research (Schwarz et al. 1991b; Wänke et al. 1996) manipulated the subjective experience that accompanies actual retrieval, the present study demonstrates that imagined experience can lead to identical effects. Experienced as well as imagined difficulty in information retrieval served to discount the implications of information content. An ad that employed recipients' self-generation of product benefits resulted in a more favorable attitude toward the target brand and a more unfavorable attitude toward the competitor brand when recipients either experienced or merely imagined this generation task as easy. An ad that asked recipients to retrieve negative brand information resulted in a more favorable attitude toward the target brand and a more unfavorable attitude toward a competitor brand when this retrieval task was difficult rather than easy.

\section{Possible Mediators}

As was mentioned in the introduction, there are several possible mediators of the impact of ease of retrieval (for a more detailed account, see Wänke et al. [1996]). According to the availability heuristic (Tversky and Kahneman 1982), individuals infer a scarcity of pro arguments and arrive at a less positive evaluation if the retrieval of pro-BMW arguments is difficult rather than easy. Alternative accounts suggest that difficulty may reduce the perceived diagnosticity of the information. Whether the notion of difficulty is experienced personally or merely inferred from other sources should not affect its impact on subsequent judgments.

The present study does not allow us to distinguish whether or not the observed effects are due to imagined failure rather than imagined difficulty. However, in the absence of an actual experience and actual outcome, individuals may not distinguish between success with difficulty and failure. After all, whether one succeeds with difficulty or does not succeed at all is mainly a function of invested effort. In this respect, we argue that imagined difficulty and imagined failure are merely different facets of the same picture.

It may be argued on the one hand that failure, difficulty, or the mere thought of engaging in cognitive activity may cause negative affect. However, this would not explain the differential impact of difficulty for pro versus contra reasons. On the other hand, experience-elicited affect may have served a mediating function by affecting the processing of the information rather than directly affecting the judgments. Previous research has shown that, compared with a happy mood, a sad mood leads to more systematic processing of persuasive arguments and thus to a decreased impact of weak arguments (see Schwarz, Bless, and Bohner 1991a). Assuming that the reasons retrieved by ad recipients were not extremely compelling, negative affect may have caused less attitude change than positive affect. Unfortunately, we did not include measures of affect. Although one may assume that experiencing ease contributes to positive affect (see, e.g., Csikszentmihalyi 1975) and difficulty to negative affect, the only "ease" study of which we are aware that assessed affect found no effect of manipulated difficulty on assessed mood (Werrmann 1996). This does not preclude the emergence of mood effects in the present study, but mood effects on the processing of information would not explain the effects of anticipated experience where in fact no information was retrieved.

\section{Applied Implications}

Three aspects of our findings are especially noteworthy for applied purposes. First, our results recommend that advertisers encourage consumers to generate brand benefits only if consumers can do so easily. Should consumers experience or merely imagine difficulty, they are likely to arrive at a more negative evaluation. Second, the effects of retrieving negative product information suggest a new 
FIGURE 2

DIRECT PREFERENCES AS A FUNCTION OF AD TYPE

\section{Brand preference}

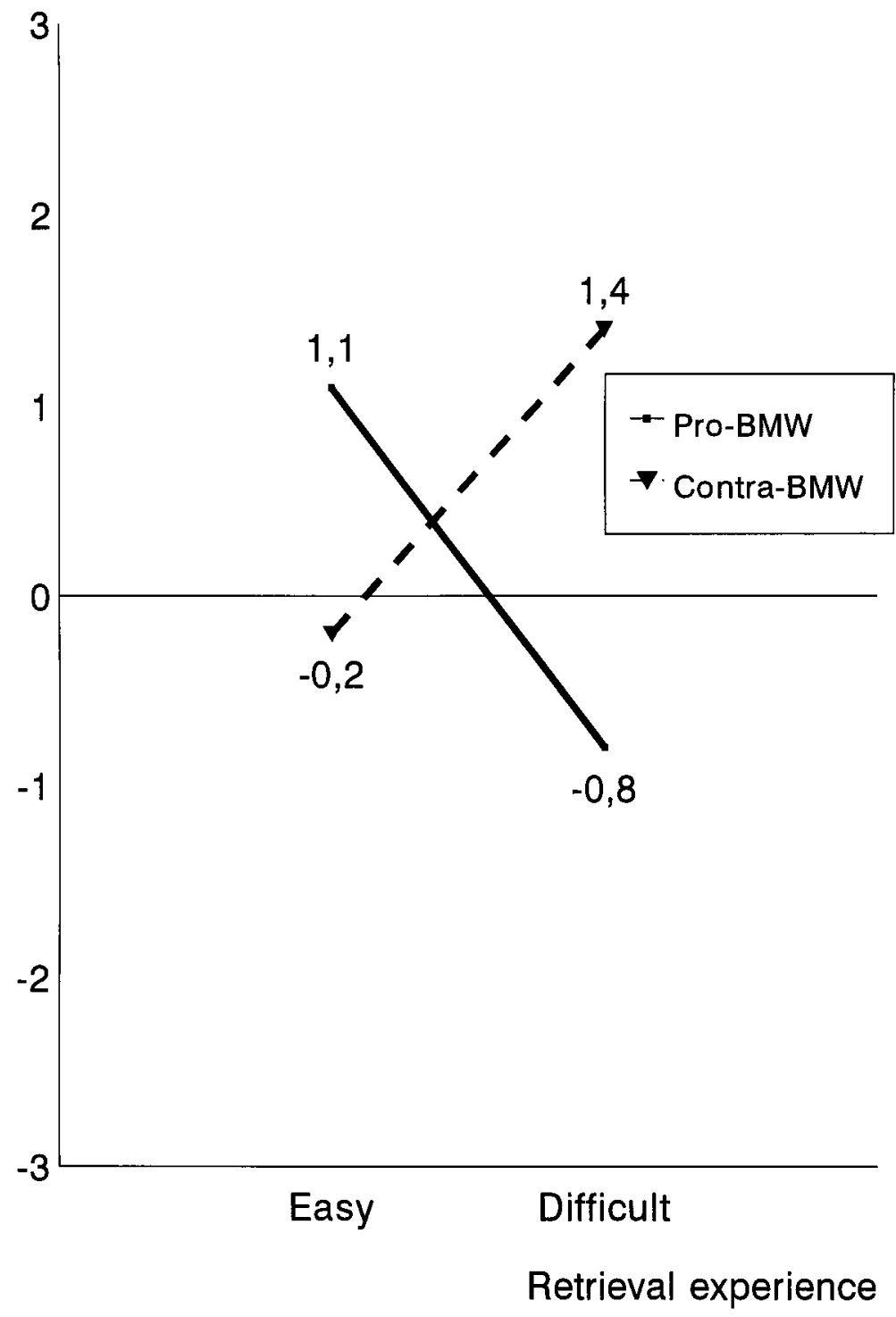

NOTE. - Positive numbers indicate a preference for BMW, and negative numbers indicate a preference for Mercedes.

strategy. Because difficulty in retrieving negative product information can result in rather positive attitudes toward a product compared with its competitor, asking consumers to retrieve negative information may provide a useful strategy for brands or products that lack both distinctively positive and negative features. Even when little positive product information can be presented, the experience of difficulty or failure in coming up with any negative information may exert a positive influence on attitudes toward the product. Third, if ad recipients do not engage in information retrieval but rather imagine their experience, this imagination process itself may be subject to external in- fluences. For example, certain cues in the situation might evoke the impression that the thought generation would be easy or difficult. At present, this assumption is still highly speculative, and further research is needed to provide evidence both for the proposed dynamics and for the nature of the cues that would trigger them.

\section{Relation to Previous Findings in the Advertising Literature}

Having ad recipients self-generate product benefits is not the only advertising strategy that employs recipients' 
active input. Other studies have shown that, at least for highly involved consumers, forming their own conclusions is more effective than being presented with conclusions (Sawyer and Howard 1991). Although many different processes may contribute to this effect, we would also suggest that the ease with which a particular conclusion springs to mind may moderate this finding. If recipients arrive at a conclusion only with difficulty, they may have less confidence in that conclusion and rely less on it (see Wänke et al. 1996).

At present, our study and other research has only investigated the effects of subjective ease in information generation on judgments assessed shortly after the information generation. Asking ad recipients to self-generate product benefits may, however, also prove to be an effective strategy because self-generated information is recalled better than externally presented information (Slamecka and Graf [1978]; see Moore, Reardon, and Durso [1986] for research in an advertising context).

\section{CONCLUSIONS}

We suggest that the experienced and the imagined subjective experience with which ad recipients process brand information is an important factor in determining recipients' brand attitudes. Both actual and imagined experience may thus be worthwhile targets of brand or product communication. More generally, our results confirm earlier findings on the mediating role of experienced ease in attitude change. In addition, they complement the earlier findings insofar as merely imagined as opposed to experienced ease is a sufficient mediating variable. Although the processes underlying the impact of subjective experience are not yet fully understood, we believe that our findings offer insights for the theoretical understanding of ease effects and for their application in the advertising context.

[Received February 1995. Revised January 1997. Brian Sternthal served as editor and Deborah Roedder John served as associate editor for this article.]

\section{REFERENCES}

Csikszentmihalyi, Mihalyi (1975), Beyond Boredom and Anxiety, San Francisco: Jossey-Bass.

Higgins, E. Tory (1989), “'Knowledge Accessibility and Activation: Subjectivity and Suffering from Unconscious Sources," in Unintended Thought, ed. James S. Uleman and John A. Bargh, New York: Guilford, 75-123.

Janis, Irving L. and Bert T. King (1954), "The Influence of Role-Playing on Opinion Change,' Journal of Abnormal and Social Psychology, 49, 211-218.

Kardes, Frank R. (1994), "Consumer Judgment and Decision Process," in Handbook of Social Cognition, ed. Robert S.
Wyer and Thomas K. Srull, Hillsdale, NJ: Erlbaum, 323 417.

King, Bert T. and Irving L. Janis (1956), "Comparison of the Effectiveness of Improvised versus Non-Improvised RolePlaying in Producing Opinion Change,' Human Relations, 9, 177-186.

Kisielius, Jolita and Brian Sternthal (1984), "Examining the Vividness Controversy: An Availability-Valence Interpretation," Journal of Consumer Research, 12 (March), 418431.

Moore, David J., Richard Reardon, and Francis T. Durso (1986), "The Generation Effect in Advertising Appeals," Advances in Consumer Research, 13, 117-120.

Rosenthal, Robert and Ralph L. Rosnow (1985), Contrast Analysis, Cambridge: Cambridge University Press.

Sawyer, Alan G. and Daniel J. Howard (1991), "Effects of Omitting Conclusions in Advertisements to Involved and Uninvolved Audiences,' Journal of Marketing Research, 28 (November), 467-474.

Schwarz, Norbert, Herbert Bless, and Gerd Bohner (1991a), "Mood and Persuasion: Affective States Influence the Processing of Persuasive Communications,"' In Advances in Experimental Social Psychology, Vol. 24, ed. M. Zanna, San Diego, CA: Academic Press, 161-199.

, Herbert Bless, Fritz Strack, Gisela Klumpp, Helga Rittenauer-Schatka, and Anette Simons (1991b), "Ease of Retrieval as Information: Another Look at the Availability Heuristic,' Journal of Personality and Social Psychology, 61 (February), 195-202.

Slamecka, Norman J. and Peter Graf (1978), "'The Generation Effect: Delineation of a Phenomenon,' Journal of Experimental Psychology: Human Learning and Memory, 4 (6), 592-604.

Strack, Fritz, Norbert Schwarz, and Elisabeth Gschneidinger (1985), "Happiness and Reminiscing: The Role of Time Perspective, Mood, and Mode of Thinking," Journal of Personality and Social Psychology, 49 (6), 1460-1469.

Tversky, Amos and Daniel Kahneman (1982), "Availability: A Heuristic for Judging Frequency and Probability,' in Judgment under Uncertainty: Heuristics and Biases, ed. Daniel Kahneman et al., Cambridge: Cambridge University Press, 163-178.

Wänke, Michaela, Herbert Bless, and Barbara Biller (1996), "Subjective Experience versus Content of Information in the Construction of Attitude Judgments," Personality and Social Psychology Bulletin, 22 (November), 1105-1113.

- and Claudia Gierke (1995), "Assimilation and Contrast in Consumer Satisfaction: When Likes Result in Lower Satisfaction than Dislikes,' paper presented at the Association for Consumer Research Conference, Minneapolis.

- Norbert Schwarz, and Herbert Bless (1995), "The Availability Heuristic Revisited: Experienced Ease of Retrieval in Mundane Frequency Estimates,' Acta Psychologica, 89, 83-90.

Werrmann, Sabine (1996), "Persuasive Kommunikation und Einstellungsänderung: Zum Zusammenwirken von Kommunikationsinhalt, subjektiver Erfahrung und Motivation,' unpublished diploma thesis, Abteilung Sozialpsychologie, Universität Heidelberg, 69117 Heidelberg.

Wyer, Robert S. and Thomas K. Srull (1989), Memory and Cognition in Its Social Context, Hillsdale, NJ: Erlbaum. 